\title{
Correction to: Quality of life in bladder cancer patients receiving medical oncological treatment; a systematic review of the literature
}

\author{
G. A. Taarnhøj ${ }^{1}$, C. Johansen ${ }^{1,2}$ and H. Pappot ${ }^{*}$
}

\section{Correction to: Health Qual Life Outcomes (2019);17:20 https://doi.org/10.1186/s12955-018-1077-6}

The original article [1] contains a major error whereby a main Table is omitted. Thus, new Table 3 (shown ahead) should be considered as Table 3 in the original article. All citations in the text to tables are with these changes are correct.

\section{Author details}

'Department of Oncology, Rigshospitalet, Blegdamsvej 9, section 5073, 2100 Copenhagen $\varnothing$, Denmark. ${ }^{2}$ Unit of Survivorship, Danish Cancer Society,

Strandboulevarden 49, 2100 Copenhagen $\varnothing$, Denmark.

Published online: 23 January 2020

\section{Reference}

1. Taarnhøj GA, Johansen C, Pappot H. Quality of life in bladder cancer patients receiving medical oncological treatment; a systematic review of the literature. Health Qual Life Outcomes. 2019;17:20. https://doi.org/10.1186/ s12955-018-1077-6.

Full list of author information is available at the end of the article 
Table 3 Outcome: Overall QoL

\begin{tabular}{|c|c|c|c|c|c|c|c|}
\hline & & GRADE issue & & & & & Overall GRADE rating \\
\hline & $\mathrm{Nb}$. of studies & Risk of bias & Inconsistency & Indirectness & Imprecision & Publication bias & \\
\hline Before diagnosis & $1[12]$ & $x^{1}$ & & $x^{2}$ & $x^{3}$ & & $\oplus \oplus \bigoplus \oplus$ \\
\hline Before treatment & $4[14,18-19,27]$ & & $x^{4}, x^{5}$ & & & & $\oplus \oplus \oplus \oplus$ \\
\hline During treatment & $2[16,18]$ & & $x^{4}$ & $x^{6}$ & $x^{3}$ & & $\bigoplus \oplus \bigoplus \bigoplus$ \\
\hline $\begin{array}{l}\leq 6 \text { months after } \\
\text { treatment }\end{array}$ & $4[18-19,27,34]$ & $x^{7}$ & $x^{5}$ & & & & $\oplus \oplus \oplus \oplus$ \\
\hline $\begin{array}{l}>6 \text { months after } \\
\text { treatment }\end{array}$ & $7[13,20-21,24-25,27,35]$ & $x^{8}$ & $x^{9}, x^{5}$ & & & None apparent ${ }^{10}$ & $\oplus \oplus \oplus \oplus$ \\
\hline
\end{tabular}

${ }^{1}$ Two validated QoL questionnaires with very large difference between outcomes

${ }^{2}$ Predominantly NMIBC patients, scores not specific for MIBC population

${ }^{3}$ Small number of patients

${ }^{4}$ Large difference in populations hence diverging scores

${ }^{5}$ Use of different QoL scales

${ }^{6}$ Only one study representing metastatic population, no studies representing neoadjuvant population

${ }^{7}$ Mixed BC population, selected population [34]

${ }^{8}$ Compliance $47-77 \%$, thereby introducing selection bias

${ }^{9}$ QoL questionnaires designed for prostate population

${ }^{10}$ As estimated by funnel plot 